\title{
Project Index
}

Bold type numbers mean that the given project is also mentioned or discussed on the following page(s) of the same paper.

AGAPE 10

ASAS 10, 19

COROT $41,69,74$

DIRECT 10, 19, 182

DIVA 42

DMT 14

ELODIE $269, \mathbf{2 8 6}$

EROS $\mathbf{9}, 26,83, \mathbf{1 3 9}, \mathbf{1 9 4}, 249, \mathbf{2 5 0}, 334$

FAME 42,76

GAIA 41,71

GONG 419

Hipparcos $42, \mathbf{4 6}, \mathbf{6 0}, \mathbf{6 2}, 71,77, \mathbf{1 0 1}, \mathbf{1 3 3}, \mathbf{2 1 2}, 237,265,277,402, \mathbf{4 3 4}, 436$, 449,532

HST $14, \mathbf{3 8}, 486,54,82,145, \mathbf{1 8 4}, 201,207,216, \mathbf{2 2 9}, \mathbf{2 3 1}, 246$

ILMT 71

IUE 246

Kepler 45

LOTIS 10

MACHO $\mathbf{9}, 26,30,72,78,83, \mathbf{8 9}, \mathbf{1 1 4}, 120, \mathbf{1 7 2}, 194,207,249,273,276, \mathbf{2 9 1}$, $299, \mathbf{3 1 3}, 334$

2MASS 16

MEGA 10

MOA $10,31,78,80,120$

MONS 44,73

MOST 44,74

NPOI 152

OGLE $\mathbf{9}, \mathbf{1 9}, 26,30,51,72,100,194,203, \mathbf{2 0 8}, 249, \mathbf{2 5 0}, 254,275,276,314$, $\mathbf{3 1 5}, 334$

PLANET 10, 25

ROSAT 516

ROTSE 10

SDSS $16,68,76,514$

SIM 77

STACC $419,471,473$

STARE 10

STEPHI $419, \mathbf{4 7 7}, \mathbf{4 8 1}, \mathbf{4 8 3}$

Tycho 62

WET 419,523 